\title{
Hacia el manejo de ecosistemas forestales
}

\author{
Oscar A. Aguirre Calderón ${ }^{1}$
}

\begin{abstract}
RESUMEN
El manejo forestal tradicional tiende hacia el manejo de ecosistemas forestales, esto es, hacia el logro y mantenimiento de la condición ecológica y forestal deseadas, dentro de las cuales se consigue el rendimiento sostenido de productos para satisfacer necesidades humanas.

El desarrollo de modelos de manejo de ecosistemas es una tarea compleja que involucra la participación de diversos sectores de la sociedad, así como de equipos multidisciplinarios de profesionales para la toma de decisiones. El objetivo es lograr la mayor interacción entre necesidades sociales, necesidades económicas y capacidades de los ecosistemas.
\end{abstract}

En este artículo se presentan algunos principios generales de manejo de ecosistemas forestales, así como la problemática que implica su puesta en práctica, destacando aspectos diferenciales con el manejo forestal tradicional. Se mencionan asimismo diversas estrategias de manejo a nivel de paisaje y para rodales individuales, para contribuir al manejo holístico de los recursos forestales.

PALABRAS CLAVE:

Desarrollo de modelos, manejo de ecosistemas forestales, manejo forestal, paisaje forestal.

\begin{abstract}
Traditional forest management is giving way to forest ecosystem management. This transition leads towards achievement and sustainability of the desired ecological and forest conditions, obtaining a sustained yield of products for the satisfaction of human needs.
\end{abstract}

The development of forest ecosystem management models is a complex task, which involves the participation of several society sectors, as well as interdisciplinary professional teams for decision making. The objective is to achieve the greatest interaction between social requirements, ecological needs and ecological capabilities.

In this article some principles of forest ecosystem management are presented, as well as the issues that running such a system implies, pointing out different aspects from traditional forest management. Several strategies for management forest landscape and forest stands, in order to contribute to a holistic forest resource management, are discussed.

KEY WORDS:

Model development, forest ecosystem management, forest management, forest landscape.

1 Facultad de Ciencias Forestales, Universidad Autónoma de Nuevo León. Apdo. Postal 41. 67700 Linares, Nuevo León, México.

Manuscrito recibido para su publicación el 4 de Julio de 1996 
El manejo de recursos forestales tiene como base el concepto de rendimiento sostenido, que se refiere a la capacidad de las áreas boscosas de proveer de manera permanente y óptima los múltiples bienes y servicios que la sociedad demanda. Aunque este concepto contempló en sus orígenes el manejo de recursos forestales de manera holística (Schanz, 1996), la evolución del mismo en los últimos dos siglos ha orientado principalmente a la generación de esquemas de producción sostenible de bienes tangibles o con valor de mercado, principalmente madera. En este marco, el manejo forestal tradicional, entendido como una secuencia de decisiones encaminadas a proveer permanentemente a la sociedad de un flujo de productos, minimizando efectos ambientales adversos, ha desarrollado modelos de gestión de los recursos que, si bien han elevado los niveles de producción de las áreas forestales, esto no se ha dado siempre bajo consideración del efecto de las técnicas aplicadas sobre la totalidad de los elementos del ecosistema. El modelo del bosque regular normal ilustra en buena medida el objetivo del manejo forestal tradicional y su orientación hacia la obtención de bienes.

En la última década, la comunidad internacional ha establecido una serie de estrategias de manejo de recursos naturales bajo el concepto de desarrollo sustentable (Earth Summit, 1992). En este marco, el manejo forestal tradicional enfrenta actualmente el reto de generar esquemas que garanticen el mantenimiento de la diversidad biológica y la fertilidad del suelo, la conservación de la variabilidad genética y su dispersión, así como todas las funciones ecológicas de las áreas bajo manejo. En síntesis, el manejo forestal tradicional debe tender hacia el manejo de ecosistemas forestales, es decir, al logro y mantenimiento de la condición ecológica y forestal deseadas, dentro de

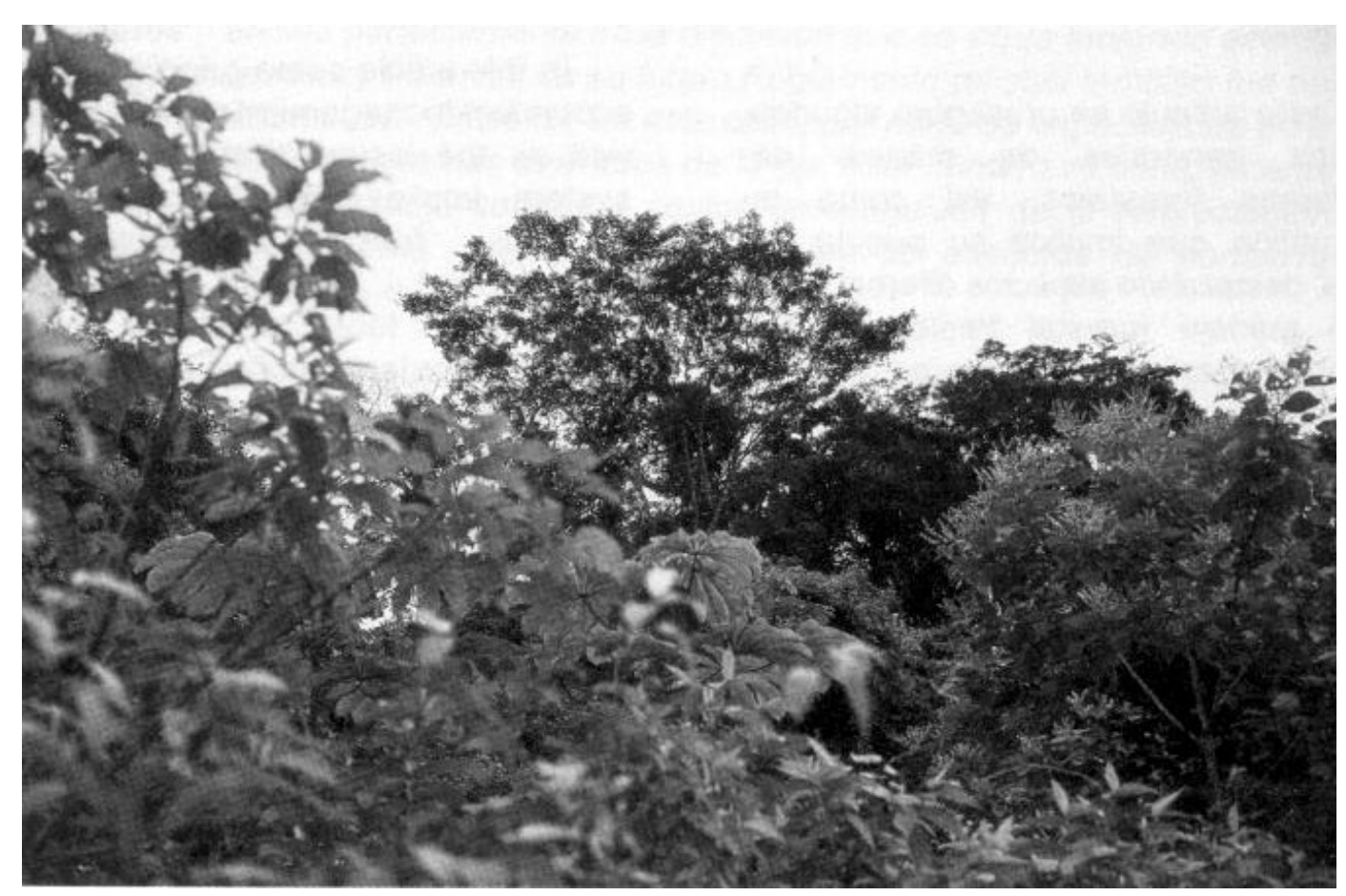


las cuales se consigue el rendimiento sostenido de productos para satisfacer necesidades humanas. El manejo de ecosistemas forestales debe entenderse entonces como un concepto del manejo de recursos naturales, en el que las actividades forestales se consideran en el marco de las interacciones ecológicas, económicas y sociales dentro de un área o región determinada, a corto y largo plazo (Thomas, 1996).

Aunque el manejo forestal tradicional aplica principios ecológicos a la gestión de los recursos, su diferencia con el manejo de ecosistemas es probablemente el enfoque desde el cual se interactúa con las áreas boscosas. Mientras que el manejo tradicional atiende básicamente la densidad de recursos (volumen de madera, densidad de fauna, infraestructura, etc.), así como el rendimiento de los mismos; el manejo de ecosistemas enfatiza sobre la condición o estado forestal, evaluando de manera adicional o con mayor detalle parámetros como : diversidad biológica, tipo de paisaje, productividad del suelo, edad, estructura, vigor, composición florística, fauna, residuos maderables, etc. Desde este punto de vista, ambos procedimientos de gestión no son de ninguna manera contrapuestos, sino complementarios. El manejo de ecosistemas no excluye la producción de bienes de mercado en las áreas forestales, simplemente no permite que la obtención de un producto dado sea el único objetivo de manejo. La producción de madera, por ejemplo, debe ganar su lugar en el conjunto de bienes y servicios, justo como cualquier otro producto del bosque de uso activo o pasivo (Hunter, 1990).

El manejo de ecosistemas forestales contempla la toma de decisiones sobre la gestión de los recursos en dos horizontes: uno temporal y uno espacial. La finalidad es lograr la mayor interacción posible entre necesidades sociales, necesidades económicas y capacidades ecológicas; procurando un balance entre las demandas de productos y servicios a corto plazo y las demandas de persistencia a largo plazo.

En el horizonte temporal se plantean estrategias a diferentes plazos con el objetivo de alcanzar la condición forestal deseada, esto es, se parte de una condición original o inicial y se generan modelos de manejo para avanzar hacia la condición objetivo, o de referencia. En el horizonte espacial, se proponen básicamente esquemas a nivel de paisaje (filtro grueso o procedimiento macro) y para rodales individuales (filtro fino 0 procedimiento micro).

Conviene destacar que los modelos de manejo de ecosistemas forestales tienen, como el modelo del bosque normal, un importante grado de idealidad. Lo que hoy concebimos como un modelo de gestión adecuado para el largo plazo, en las próximas décadas o siglos probablemente pierda vigencia. Sin embargo, tal como el modelo del bosque normal (con sus limitaciones, por supuesto) ha orientado las acciones de manejo con el objeto de conservar los recursos forestales, los nuevos procedimientos de gestión deben entenderse como la meta hacia la cual dirigir las acciones, considerando siempre que lograr la condición deseada en un paisaje forestal puede trascender varias generaciones. En este contexto, es importante considerar que el manejo de ecosistemas se debe adaptar a los nuevos paradigmas de gestión de recursos naturales, generados por la experiencia, el avance científico y el desarrollo de la sociedad (Bormann et al., 1994).

El manejo de ecosistemas no es una metodología única depurada o validada suficientemente, dada la complejidad y carácter dinámico de los ecosistemas. 


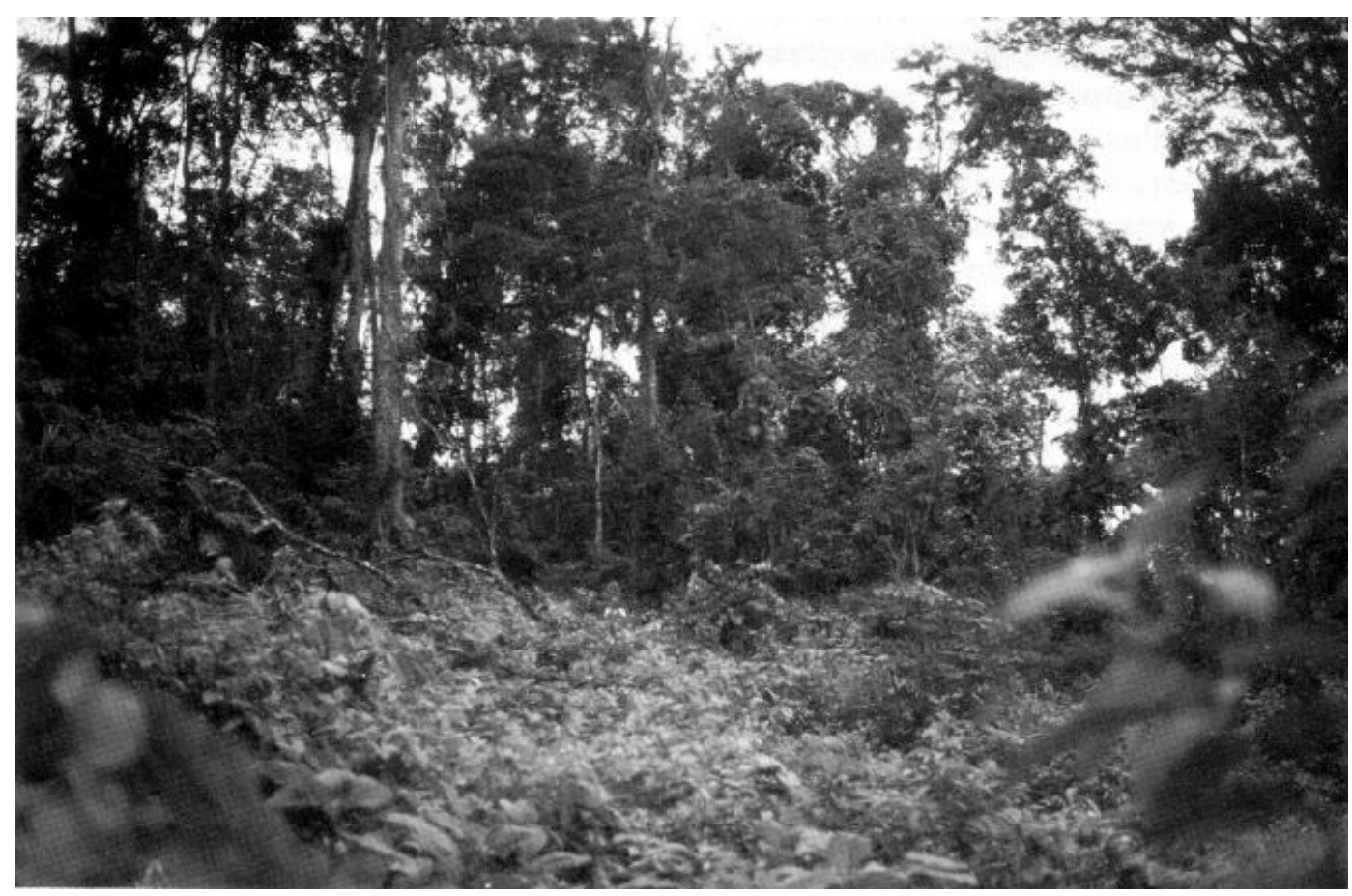

Consiste mas bien en la aplicación de una serie de herramientas para el desarrollo de modelos capaces de determinar el impacto de diversas acciones de manejo, teniendo siempre en consideración el ingrediente estocástico, tanto por el carácter de los recursos bajo manejo, como por la necesidad de describir los valores sociales para recursos bajo manejo, como por la necesidad de describir los valores sociales para recursos de mercado y no de mercado - tarea nada fácil - de manera que los conflictos se puedan manejar eficientemente en el proceso de toma de decisiones. La idea básica es entender cada acción o cambio como un efecto en un sistema complejo de procesos y evaluar tales acciones y cambios desde la perspectiva de un todo (Schlaepfer, 1997).
Un apoyo importante para el manejo de ecosistemas forestales lo constituyen los planes de ordenamiento ecológico y/o territorial a diferentes escalas. Una vez definida el área bajo manejo, tales planes permiten jerarquizar las unidades de gestión (paisaje, ecosistema, comunidad, población) e identificar la vocación de las mismas para generar el plan de manejo respectivo. Los sistemas de información geográfica y de recursos tienen gran importancia en este contexto, ya que posibilitan la evaluación de la condición original del área y el monitoreo de los resultados de las diversas acciones realizadas.

La unidad de manejo de ecosistemas forestales es el paisaje, entendido como una superficie compuesta por ecosistemas interactuantes que ocurren repetidamente 
con un patrón de distribución determinado por la geología, fisiografía, suelos, clima, biota e influencias antropogénicas. Tal circunstancia implica que puede abarcar diversos predios $y$, por ende, varios propietarios o poseedores con objetivos de manejo frecuentemente distintos a los previstos en el marco de la planeación a nivel de ordenamiento ecológico o del plan de manejo de ecosistemas. Lo anterior implica que para lograr la aplicación de estrategias de gran visión, debe existir un acuerdo entre los sectores interesados y, eventualmente, generar un esquema de incentivos $y / 0$ estímulos para quienes deben renunciar a la realización de una actividad productiva o se ven afectados por la disminución del nivel de rendimiento de productos. Lograr acuerdos en tal sentido requiere de la participación de los diversos sectores involucrados, con el fin de atender de manera balanceada la problemática ecológica, social y económica del área en cuestión (Kaufmann, 1994).

La complejidad del manejo de los ecosistemas forestales obliga necesariamente a la integración de equipos multidisciplinarios y a una ampliación del enfoque reduccionista que priva aún en diversas profesiones, tanto para la generación de conocimientos que permitan apoyar la toma de decisiones, como para el manejo de los recursos. Por otra parte, las necesidades de conocimiento científico en ciencias biológicas, físicas y económicas, así como en aspectos políticos y sociales, constituyen un reto para profesionales de diversas disciplinas, responsables de la gestión de los ecosistemas forestales.

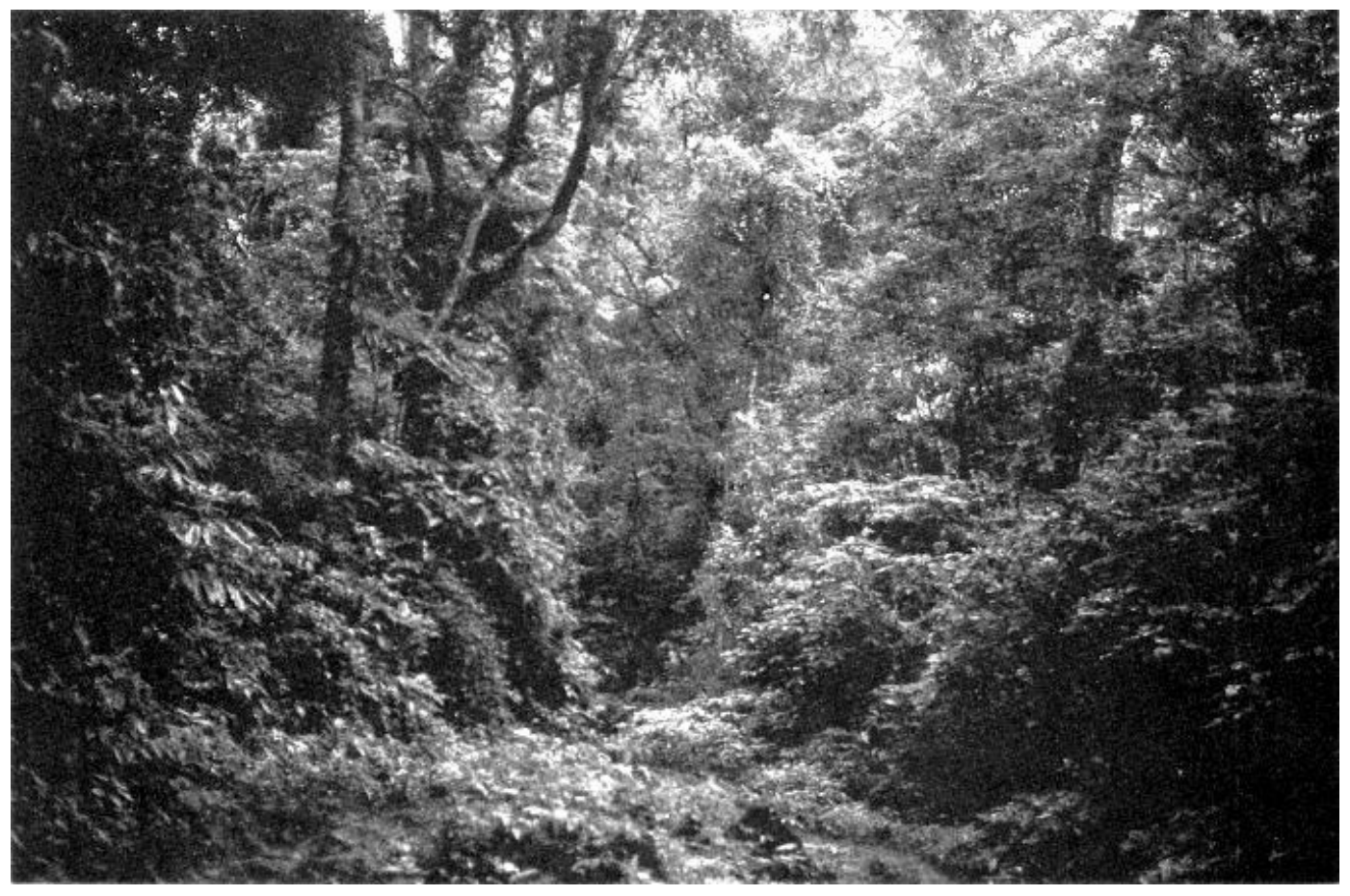




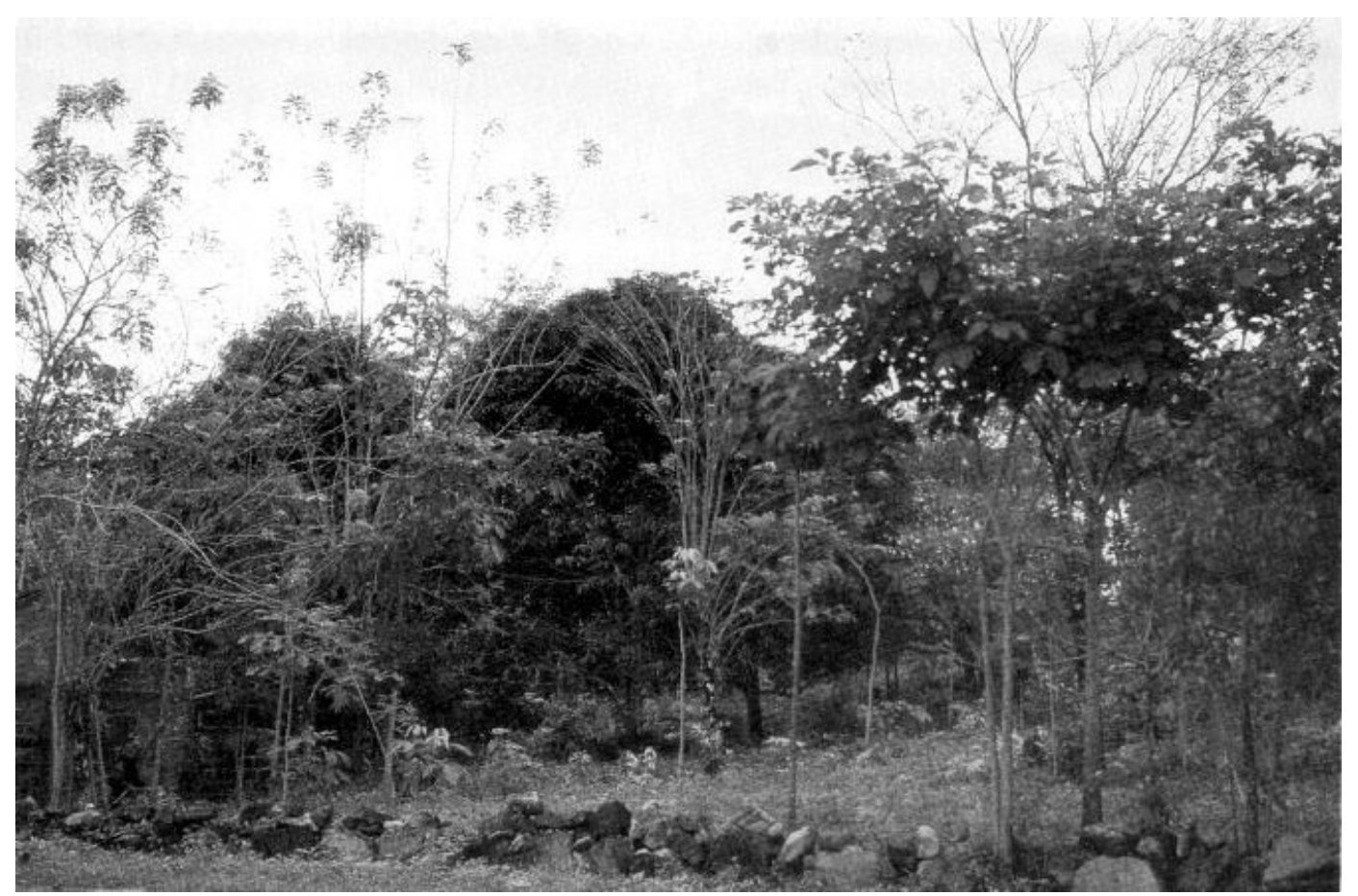

En un esfuerzo por integrar modelos de gestión holísticos, los diferentes sectores involucrados aportan continuamente elementos que contribuyen a este propósito. Dentro de los aspectos sobre los que la comunidad forestal internacional ha puesto especial atención hacia el manejo de ecosistemas forestales destacan los que se mencionan en los siguientes párrafos. Los tópicos incluidos comprenden fundamentalmente prácticas de carácter silvícola enfocadas a la conservación y fomento de la biodiversidad, que pueden encaminar el proceso de gestión hacia la condición forestal deseada, esto es, hacia un ecosistema de referencia (Hunter, 1990; Hatzfeldt, 1994; Thomasius y Schmidt, 1996; Scherzinger, 1996).

En el marco del manejo de paisajes forestales, la composición de especies ha cobrado particular relevancia, la tendencia es mantener o restaurar la composición específica natural de los rodales. Cuando los objetivos económicos conllevan a un cambio de especies en determinadas áreas, la elección de dos o más especies nativas para el establecimiento de rodales mixtos puede ser una estrategia adecuada. No debe descartarse, sin embargo, el establecer masas puras en algunas áreas de alta productividad.

Lograr una estructura de edades balanceada a nivel de paisaje cumple dos objetivos: garantiza el rendimiento sostenido de productos forestales y proporciona un hábitat diverso para la fauna silvestre. De esta manera el manejo puede contribuir a un incremento de la biodiversidad. Es importante considerar que los bosques alcanzan la madurez económica mucho antes que la biológica, sin embargo, el mantenimiento y protección 
de bosques sobremaduros distribuidos convenientemente en el área, garantiza la preservación de la comunidad como tal y de las especies asociadas a la misma.

En la determinación del tamaño, aspecto y disposición de los rodales, se destaca la importancia de considerar las características de los límites o bordes en el paisaje, producto de las decisiones de manejo. En este sentido, deben tomarse en cuenta básicamente el grado de mezcla de los ecosistemas a fin de garantizar la provisión de hábitat a las especies asociadas a áreas núcleo, límites y ecotonos.

Un aspecto de particular importancia a nivel de paisaje es el lograr una heterogeneidad espacial adecuada. Esta se refiere básicamente a contar con unidades de manejo de diferentes superficies y manejadas bajo criterios distintos en lo que a sistema silvícola, turno y estructura se refiere, de manera tal que se logren objetivos ecológicos y económicos satisfactoriamente. Bajo un esquema de distribución adecuado pueden incluírse, por ejemplo, áreas de matarrasa y áreas de selección contiguas.

En las franjas adyacentes a las corrientes de agua, el esquema puede basarse en la delimitación de una franja libre de corta cercana a la corriente, seguida de una zona más amplia de corta de baja intensidad.

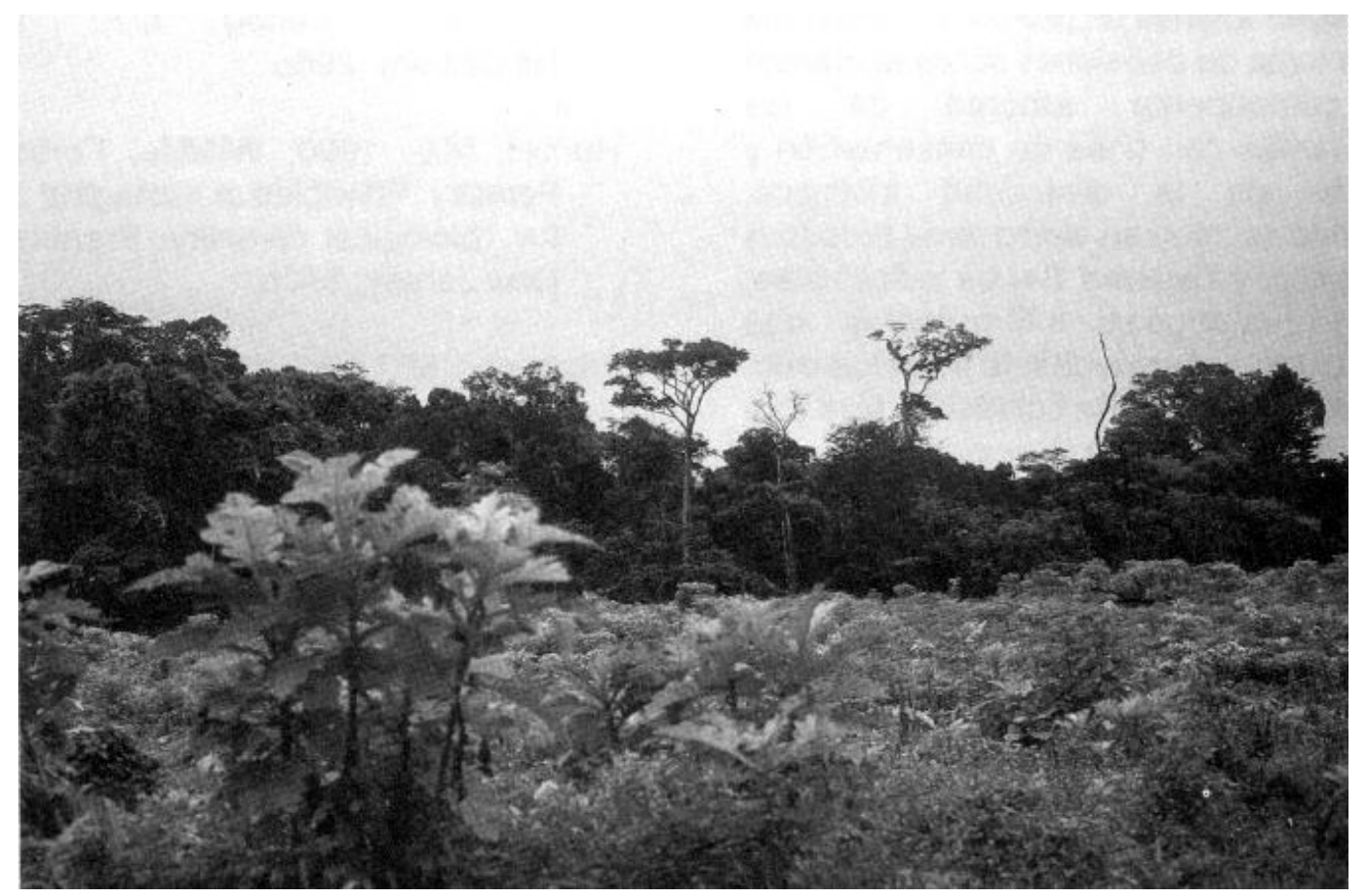


A nivel de rodal, las estrategias de manejo se enfocan en mayor medida a la conformación de una estructura vertical que conforman masas irregulares. No todos los rodales pueden ni deben tener una estructura vertical bien desarrollada, pero es importante que aquellos multicohortales se encuentren adecuadamente distribuidos a través del paisaje. Una buena estructura vertical se logra también plantando fracciones del rodal en diferentes etapas a nivel de individuos, grupos o franjas.

Finalmente, cabe destacar la importancia de mantener árboles moribundos, muertos y caídos de grandes dimensiones para preservar las especies asociadas a los mismos. Tales árboles pueden ubicarse distribuidos en el rodal de forma individual, o en una fracción del mismo formando bosquetes sobremaduros.

Las prácticas antes enlistadas constituyen apenas un marco de referencia para la toma de decisiones sobre el manejo del componente arbóreo de los ecosistemas con fines de conservación y fomento de la diversidad biológica. Conforme se integran al esquema procesos ecológicos y factores físicos adicionales, surgen numerosas interrogantes que representan necesidades de investigación. La determinación de la condición deseable o de referencia de los ecosistemas, los procedimientos de inventario de su condición actual, las consecuencias de diversas opciones de manejo, la evaluación y modelaje de procesos de sucesión y disturbio, los efectos de la introducción de especies exóticas y el desarrollo de métodos de monitoreo de los cambios en los ecosistemas son, entre muchos otros, conocimientos que deberán integrarse en la generación de esquemas sustentables de manejo, que incluyan demás consideraciones económicas y sociales, esto es, la dimensión humana del problema.

\section{REFERENCIAS}

Bormann, B.T., M.H. Brookes, E.D. Ford, A.R. Kiester, C.D. Oliver y J.F. Weingand. 1994. A framework for sustainable ecosystem management. General Technical Report PNW-331. US Department of Agriculture, Forest Service. Pacific Northwest Research Station. Portland, OR. 61p.

Earth Summit, 1992: Agenda 21. The United Nations Programme of action from Rio. Final text of agreements negotiated at the United Nations Conference of Environmental and Development (UNCED). June 3-14, 1992. Rio de Janeiro, Brazil. United Nations Department of Public Information. New York. 294p.

Hatzfeld, H.G. 1994. Ökoligische Waldwirtschaft. Grundlagen - Aspekte Beispiele. Verlag C.F. Müller. Heidelberg. 296p.

Hunter, M.L. 1990. Wildlife, Forest and Forestry. Principles of managing forests for biological diversity. Prentice-Hall. New Jersey. 370p.

Kaufmann, M.R., R.T. Graham, D.A. Boyce, W.P. Moir, L. Perry, R.T. Reynolds, R.L. Basset, P. Melhop, C.B. Edminster, W.M. Block y P.S. Corn. 1994. An ecological basis for ecosystem management. General Technical Report RM-246. US Department of Agriculture. Forest Service. Rocky Mountain Forest and Range Experiment Station. Fort Collins, CO. 22p

Schanz, H. 1996. Forstliche Nachhaltigkeit. Sozialwissenschaftliche Analyse der Begriffsinhalte und-funktionen. Schriften aus dem Institut für Fdorstökonomie der Universität Freiburg. Band 4. Freiburg. $131 p$. 
Scherzinger, W. 1996. Naturschutz mi Wald. Qualitätsziele einer dynamischen Waldentwincklung. Verlag Eugen Ulmer. Stuttgart. 442p.

Schlaepfer, R. 1997. Ecosystem-based management of natural resources. IUFRO Ocassional Paper No. 6. Viena.

Thomas H. 1996. The forest service approach to healthy ecosystems. Journal of Forestry 94(8):14-18.

Thomasius, H. y P.A. Schmidt. 1996. Wald, Forstwirtschaft und Umwelt. Economica Verlag. Bonn. 53p. $32 p$. 